\title{
Sampling of compact signals in offset linear canonical transform domains
}

\author{
Adrian Stern
}

Received: 26 February 2007 / Revised: 21 June 2007 / Accepted: 22 June 2007 / Published online: 19 July 2007

(C) Springer-Verlag London Limited 2007

\begin{abstract}
The offset linear canonical transform (OLCT) is the name of a parameterized continuum of transforms which include, as particular cases, the most widely used linear transforms in engineering such as the Fourier transform (FT), fractional Fourier transform (FRFT), Fresnel transform (FRST), frequency modulation, time shifting, time scaling, chirping and others. Therefore the OLCT provides a unified framework for studying the behavior of many practical transforms and system responses. In this paper the sampling theorem for OLCT is considered. The sampling theorem for OLCT signals presented here serves as a unification and generalization of previously developed sampling theorems.
\end{abstract}

Keywords Regular sampling - Offset linear canonical transform - Special affine Fourier transform - Linear canonical transform - Time-frequency representation . Uncertainty principle

\section{Introduction}

The sampling process is central in almost any domain because it provides the link between the continuous physical signals and the discrete time domain. The most common sampling theorem is probably the Shannon sampling theorem [1] appealing to signals of compact support in the Fourier domain. In this work we generalize Shannon's sampling theorem to signals with a compact support in the OLCT domain.

\footnotetext{
A. Stern $(\varangle)$

Department of Electro Optics Engineering,

Ben Gurion University of the Negev,

Beer-Sheva 84105, Israel

e-mail: stern@bgu.ac.il
}

The OLCT [2] is introduced in Sect. 2. The OLCT, also called special affine Fourier transform [3], is a time-shifted and frequency-modulated version of the linear canonical transform (LCT) $[2,4-6]$. Therefore it is more general and flexible than the LCT. It is a class of integral transforms that provides a canonical formalism for the response of a very large class of physical systems. Many operations, such as the FT, FRFT [7,8], FRST [9], offset FT [10], LCT, GaussWeierstrass transform [6], time shifting and scaling, frequency modulation, pulse chirping, Lorentz squeezing [3] and others, are special cases of the OLCT. Any linear combination or concatenation of the special cases of the OLCT yields also an OLCT. Thus, by developing a sampling theorem for OLCT we obtain a unified sampling theorem for all above mentioned transforms.

The condition for uniform sampling of signals with compact support in the OLCT domain is derived in Sect. 3 and the respective interpolation formula is given. This sampling theorem generalizes previously developed sampling theorems such as Shannon's sampling theorem [1], sampling theorems for signals bandlimited in the FRFT domain [1114], in the FRST domain [15], and in the LCT domain [16]. It can be viewed as a special case of Kramer's sampling theorem [17] for linear integral transforms. However, it is much more explicit and therefore, is more practical. One practical implication of the presented theorem is that it provides the sampling conditions for the output of any system that has a response described by the OLCT and that is stimulated by a bounded support input.

It is very instructive to understand the OLCT via its operation in the time-frequency (phase space) domain. The OLCT has a simple geometrical manifestation in the time-frequency domain; it performs an inhomogeneous affine mapping of the Wigner-Ville distribution (WVD) $[18,19]$ of the signal. Based on this fact, we present in Sect. 4 a heuristically and 
insightful interpretation of the OLCT sampling theorem in the time-frequency domain.

The theorem presented here generalizes and extends the sampling theorem presented in [16]. The novelty of the theorem here over that in [16] is fivefold. First, the sampling theorem presented here can be applied to a larger class of signals. For instance, in Sect. 6 is shown an example of a type of signal for which the LCT sampling theorem [16] cannot be applied directly, whereas the OLCT can be. In order to apply the LCT sampling theorem [16] to such a signal, the signal needs to be preprocessed. Second, we provide here the interpolation formula which permits direct reconstruction in the sampled domain. The reconstruction in [16] is carried out in the LCT reciprocal domain. Third, we present here an additional version of the sampling theorem that uses the properties of the OLCTed signal in the timefrequency domain. In order to use this version of the sampling theorem one needs not be familiar either with the LCT or the OLCT. Fourth, the theorem presented here holds for signals in Hilbert space $L_{C}^{2}(\Re)$, which consists of all squareintegrable signals (in Lebesgue sense), whereas the LCT in [16] requires the signals to be absolute-integrable so that their inverse LCT integral formula holds everywhere. Fifth, we present a generalization of the Heisenberg-Weyl uncertainty principle to include OLCT pairs.

\section{The OLCT}

The OLCT with real parameters $\mathbf{A}=\left(a, b, c, d, y_{0}, \omega_{0}\right)$ of a function $f(x)$ is defined by:

$\tilde{f}^{\mathbf{A}}(y)= \begin{cases}\int_{-\infty}^{\infty} f(x) h_{\mathbf{A}}(x, y) \mathrm{d} x & b \neq 0, \\ \sqrt{d} \mathrm{e}^{j \frac{c d}{2}\left(y-y_{0}\right)^{2}+j \omega_{0} y} f\left[d\left(y-y_{0}\right)\right] & b=0,\end{cases}$

where

$$
\begin{aligned}
& h_{A}(x, y)=K_{A} \mathrm{e}^{\frac{j}{2 b}\left[a x^{2}+2 x\left(y_{0}-y\right)-2 y\left(d y_{0}-b \omega_{0}\right)+d y^{2}\right]}, \\
& K_{\mathbf{A}}=\sqrt{\frac{1}{j 2 \pi|b|} \mathrm{e}^{j \frac{d y_{0}^{2}}{2 b}},}
\end{aligned}
$$

and $a d-c b=1$. The definition for case $b=0$ is the limit of the integral in (1) for the case $b \neq 0$ as $|b| \rightarrow 0$ [6]. Some of the special cases of the OLCT are listed in Table 1. Those relations can be easily verified by substituting the specific parameters $\mathbf{A}$ in Eq. (2).

For later use we note that the inverse of an OLCT with parameters $\mathbf{A}=\left(a, b, c, d, y_{0}, \omega_{0}\right)$ is given (up to a normalization factor) by an OLCT with parameters $\mathbf{A}^{-1}=$ $\left(d,-b,-c, a, b \omega_{0}-d y_{0}, c y_{0}-a \omega_{0}\right)$ [2]. This can be verified by using the definition (1) or can be easily derived from the time-frequency interpretation of the OLCT (see Sect. 4.2).
Table 1 Some of the specific cases of the OLCT and their sampling conditions

\begin{tabular}{lll}
\hline Transform & $A$ parameters & $\begin{array}{l}\text { Maximum sampling } \\
\text { interval of } \tilde{f}^{\mathbf{A}}(y)\end{array}$ \\
\hline $\begin{array}{l}\text { Offset linear canonical } \\
\text { transform (OLCT) }\end{array}$ & $(a, b, c, d, e, f)$ & $\frac{2 \pi}{B_{x}}|b|$ \\
$\begin{array}{l}\text { Fourier transform (FT) } \\
\text { Fractional Fourier }\end{array}$ & $(0,1,-1,0,0,0,0)$ & $\frac{2 \pi}{B_{x}}$ \\
transform (FRFT) & $\cos \varphi, 0,0)$ & $\frac{2 \pi}{B_{x}}|\sin (\varphi)|$ \\
Fresnel transform & $(1, b, 0,1,0,0)$ & $\frac{2 \pi}{B_{x}}|b|$ \\
(FRST) & $(a, b, c, d, 0,0)$ & $\frac{2 \pi}{B_{x}}|b|$ \\
Linear canonical & & \\
transform (LCT) & $\left(1,0,0,1, x_{0}, 0\right)$ & $\Delta$ \\
Time shifting by $x_{0}$ & $\left(d^{-1}, 0,0, d, 0,0\right)$ & $\Delta / d$ \\
Time scaling & $\left(1,0,0,1,0, \omega_{0}\right)$ & $\Delta$ \\
Frequency & & \\
modulation & &
\end{tabular}

$B_{x}$ denotes the support of $f(x)$ and $\Delta$ denotes the sampling intervals of $f(x)$ (as defined in Theorem 2)

\section{The sampling theorem}

Theorem 1 The OLCT $\tilde{f}^{\mathrm{A}}(y)$ with real parameters $\mathbf{A}=$ $\left(a, b, c, d, y_{0}, \omega_{0}\right), b \neq 0$ of a function $f(x) \in L_{C}^{2}(\Re)$ with a bounded support so that $f(x)=0 x \notin\left[-\frac{B_{x}}{2}, \frac{B_{x}}{2}\right]$, can be completely recovered from its samples at points spaced $\Delta_{y} \leq \frac{2 \pi}{B_{x}}|b|$ apart, using the following interpolation formula:

$$
\begin{aligned}
\tilde{f}^{A}(y)= & \mathrm{e}^{\frac{j}{2 b}\left[\mathrm{~d} y^{2}-2 y\left(\mathrm{~d} y_{0}-b \omega_{0}\right)\right]} \sum_{n \in Z} \tilde{f}^{A}\left(n \Delta_{y}\right) \\
& \times \frac{\sin \left[\pi\left(\frac{y}{\Delta_{y}}-n\right)\right]}{\pi\left(\frac{y}{\Delta_{y}}-n\right)} \mathrm{e}^{-\frac{j}{2 b}\left[d\left(n \Delta_{y}\right)^{2}-2 n \Delta_{y}\left(\mathrm{~d} y_{0}-b \omega_{0}\right)\right]} .
\end{aligned}
$$

Proof Let us rewrite Eq. (1) for $b \neq 0$ in the form

$\tilde{f}^{\mathrm{A}}(y)=K_{\mathrm{A}} \mathrm{e}^{\frac{j}{2 b}\left[d y^{2}-2 y\left(d y_{0}-b \omega_{0}\right)\right]} G(y)$

where $G(y)$ is defined by:

$$
\begin{aligned}
G(y) & =\int_{-\infty}^{\infty} f(x) \mathrm{e}^{\frac{j}{2 b}\left(a x^{2}+2 y_{0} x\right)} \mathrm{e}^{-\frac{j}{b} y x} \mathrm{~d} x \\
& =\left(K_{\mathbf{A}}\right)^{-1} \mathrm{e}^{-\frac{j}{2 b}\left[\mathrm{~d} y^{2}-2 y\left(\mathrm{~d} y_{0}-b \omega_{0}\right)\right]} \tilde{f}^{\mathbf{A}}(y) .
\end{aligned}
$$

By expressing $\mathrm{e}^{-\frac{j}{b} y x}$ in terms of Fourier series:

$$
\begin{gathered}
\mathrm{e}^{-\frac{j}{b} y x}=\sum_{n \in Z} \frac{\sin \left[\pi\left(\frac{y}{\Delta_{y}}-n\right)\right]}{\pi\left(\frac{y}{\Delta_{y}}-n\right)} \mathrm{e}^{-j n \frac{\Delta_{y}}{b} x}, \\
\Delta_{y} \leq \frac{2 \pi}{B_{x}}|b|, \quad|x|<\frac{B_{x}}{2},
\end{gathered}
$$


and substituting in Eq. (5) we obtain:

$$
\begin{aligned}
G(y)= & \int_{-\infty}^{\infty} f(x) \mathrm{e}^{\frac{j}{2 b}\left(a x^{2}+2 y_{0} x\right)} \\
& \times \sum_{n \in Z} \frac{\sin \left[\pi\left(\frac{y}{\Delta_{y}}-n\right)\right]}{\pi\left(\frac{y}{\Delta_{y}}-n\right)} \mathrm{e}^{-j n \frac{\Delta y}{b} x} \mathrm{~d} x \\
= & \sum_{n \in Z} \frac{\sin \left[\pi\left(\frac{y}{\Delta_{y}}-n\right)\right]}{\pi\left(\frac{y}{\Delta_{y}}-n\right)} \\
& \times \int_{-\infty}^{\infty} f(x) \mathrm{e}^{\frac{j}{2 b}\left(a x^{2}+2 y_{0} x\right)} \mathrm{e}^{-j n \frac{\Delta y}{b} x} \mathrm{~d} x .
\end{aligned}
$$

The integral in (7) can be recognized as the function $G(y)$ at sample points $y=n \Delta_{y}, n \in Z$. Thus, using Eq. (4) we obtain

$$
\begin{aligned}
G(y)= & \sum_{n \in Z} \frac{\sin \left[\pi\left(\frac{y}{\Delta_{y}}-n\right)\right]}{\pi\left(\frac{y}{\Delta_{y}}-n\right)} G(y) \mid y=n \Delta_{y} \\
= & \sum_{n \in Z}\left(K_{\mathbf{A}}\right)^{-1} \tilde{f}^{A}\left(n \Delta_{y}\right) \frac{\sin \left[\pi\left(\frac{y}{\Delta_{y}}-n\right)\right]}{\pi\left(\frac{y}{\Delta_{y}}-n\right)} \\
& \times \mathrm{e}^{-\frac{j}{2 b}\left[d\left(n \Delta_{y}\right)^{2}-2 n \Delta_{y}\left(\mathrm{~d} y_{0}-b \omega_{0}\right)\right]} .
\end{aligned}
$$

Finally substitution of Eq. (8) in Eq. (4) yields the interpolation formula Eq. (3).

By interchanging the roles of $\tilde{f}^{\mathbf{A}}(y)$ and $f(x)$ in Theorem 1 and by replacing the parameter set $\mathbf{A}=(a, b, c$, $\left.d, y_{0}, \omega_{0}\right)$ with that of the inverse OLCT $\mathbf{A}^{-1}=(d,-b,-c$, $\left.a, b \omega_{0}-d y_{0}, c y_{0}-a \omega_{0}\right)$ we obtain the sampling theorem for signals band limited in the OLCT domain, i.e., with $\tilde{f}^{\mathbf{A}}(y)=0 \quad y \notin\left[-\frac{B_{y}}{2}, \frac{B_{y}}{2}\right]$. In this form, the sampling theorem resembles the common (Shannon) sampling theorem. It is easy to verify that with $\mathbf{A}^{-1}=(0,-1,1$, $0,0,0)$, defining the (inverse) Fourier, transform we obtain Shannon sampling theorem [1] with the well known interpolation formula $f(x)=\sum_{n \in Z} f\left(n \Delta_{x}\right) \frac{\sin \left[\pi\left(\frac{x}{\Delta_{x}}-n\right)\right]}{\pi\left(\frac{x}{\Delta_{x}}-n\right)}$, $\Delta_{x}=\frac{2 \pi}{B_{y}}$. The sampling condition for the specific OLCT cases FRFT, FRST and LCT are shown in the last column in Table 1 . These results can be obtained from Theorem 1 and are in concordance with those obtained in references [1116], respectively. Note that the sampling interval depends on parameter $b$. In the case of the FRFT, and in particular for the FT, this parameter is bounded $|b| \leq 1$ setting the maximum sampling interval to $\Delta_{y}=\frac{2 \pi}{B_{x}}$. This means that there is no FRFT that can be sampled at intervals larger than $2 \pi / B x$. The case is different for the FRST where the parameter $b$ may be arbitrary large and consequently the sampling interval is virtually unbounded. In other words, the sampling interval of the FRST may be arbitrary large by proper choice of parameter $b$. This can be understood from the fact that the FRST widens the effective support of the signal by an amount proportional to $b$ (see also Sect. 5). Signal widening implies spreading the information which permits decreasing the sampling density.

Theorem 1 holds for the case $b \neq 0$ (which is the more interesting case). From definition (1) it can be seen that for $b=0$ the OLCT is simply a time scaled version of $f$ multiplied by a qudrature phase function (linear chirp) $\sqrt{d} \mathrm{e}^{j \frac{c d}{2}\left(y-y_{0}\right)^{2}+j \omega_{0} y}$. Therefore, in such a case the sampling condition is dictated by that of $f(x)$, yielding the following sampling theorem:

Theorem 2 If a function $f(x) \in L_{C}^{2}(\Re)$ can be reconstructed from its samples $x=n \Delta, n \in Z$ by some formula $f(x)=\sum_{n \in Z} f(n \Delta) \varphi_{n}(x)$, where $\left\{\varphi_{n}(x)\right\}_{n \in Z}$ is an orthogonal or Reisz basis (e.g., $\varphi_{n}(x)=\operatorname{sinc}(x / \Delta-n)$ for $f(x)$ band limited in frequency domain), then its OLCT $\tilde{f}^{\mathrm{A}}(y)$ with parameters $\mathbf{A}=\left(a, b=0, c, d, y_{0}, \omega_{0}\right)$ can be fully recovered by its samples $\Delta_{y}=\Delta / d$ apart using the interpolation formula

$$
\begin{aligned}
\tilde{f}^{\mathbf{A}}(y)= & \mathrm{e}^{j \frac{\mathrm{d} c}{2}\left(y-y_{0}\right)^{2}+j \omega_{0}\left(y-y_{0}\right)} \sum_{n \in Z} \tilde{f}^{\mathbf{A}}\left(n \Delta_{y}+y_{0}\right) \\
& \times \mathrm{e}^{-j \frac{\mathrm{d} c}{2}\left(n \Delta_{y}\right)^{2}-j \omega_{0} n \Delta_{y}} \varphi_{n}\left[d\left(y-y_{0}\right)\right] .
\end{aligned}
$$

Proof From definition (1) for the OLCT with $b=0$ we may express $f(y)$ in terms of $\tilde{f}^{\mathbf{A}}(y)$ :

$f(y)=\tilde{f}^{\mathbf{A}}\left(\frac{y}{d}+y_{0}\right) \frac{1}{\sqrt{d}} \mathrm{e}^{-j \frac{\mathrm{d} c}{2}\left(\frac{y}{d}\right)^{2}-j \omega_{0}\left(\frac{y}{d}+y_{0}\right)}$,

which, taken at points $y=n \Delta_{y}, n \in Z$ yields

$f(n \Delta)=\tilde{f}^{\mathbf{A}}\left(n \Delta_{y}+y_{0}\right) \frac{1}{\sqrt{d}} \mathrm{e}^{-j \frac{\mathrm{d} c}{2}\left(n \Delta_{y}\right)^{2}-j \omega_{0}\left(n \Delta_{y}+y_{0}\right)}$,

where $\Delta_{y}=\Delta / d$. If $f$ is such that exists a decomposition $f(x)=\sum_{n \in Z} f(n \Delta) \varphi_{n}(x)$, then by substituting Eqs. (10) and (11) in the decomposition expression we obtain

$$
\begin{aligned}
& \tilde{f}^{\mathbf{A}}\left(\frac{y}{d}+y_{0}\right) \frac{1}{\sqrt{d}} \mathrm{e}^{-j \frac{\mathrm{d} c}{2}\left(\frac{y}{d}\right)^{2}-j \omega_{0}\left(\frac{y}{d}+y_{0}\right)} \\
& =\sum_{n \in Z} \tilde{f}^{\mathbf{A}}\left(n \Delta_{y}+y_{0}\right) \frac{1}{\sqrt{d}} \mathrm{e}^{-j \frac{\mathrm{d} c}{2}\left(n \Delta_{y}\right)^{2}-j \omega_{0}\left(n \Delta_{y}+y_{0}\right)} \varphi_{n}(y) .
\end{aligned}
$$

Changing the variables $y \rightarrow d\left(y-y_{0}\right)$ in Eq. (12) and rearranging yields the formula (9). 


\section{Interpretation in time-frequency domain}

4.1 The effect of the OLCT on the Wigner distribution

Let us consider the joint parameter time-frequency representation of $f(x)$ obtained through the WVD defined by $[18,19]$ :

$W_{f}\left(x, \omega_{x}\right)=\frac{1}{2 \pi} \int_{-\infty}^{\infty} f\left(x+\frac{\xi}{2}\right) f^{*}\left(x-\frac{\xi}{2}\right) \mathrm{e}^{-j \xi \omega_{x}} \mathrm{~d} \xi$

The WVD of $\tilde{f}^{\mathbf{A}}(y)$ is related to that of $f(x)$ by:

$$
\begin{aligned}
W_{\tilde{f}^{\mathbf{A}}}\left(y, \omega_{y}\right)= & W_{f}\left[d\left(y-y_{0}\right)-b\left(\omega_{y}-\omega_{0}\right),\right. \\
& \left.-c\left(y-y_{0}\right)+a\left(\omega_{y}-\omega_{0}\right)\right] .
\end{aligned}
$$

Equation (14) can be verified directly from the definitions (1) and (13) or, alternatively, it can be proved by using the respective relation for the WVD of the LCT [20] together with the fact that the OLCT can be obtained by shifting the $(a, b, c, d)$-LCT of $f(x)$ by $y_{0}$ in the time domain and modulating with $\mathrm{e}^{j y \omega_{0}}$ (i.e., $\tilde{f}^{\left(a, b, c, d, y_{0} \omega_{0}\right)}(y)=\tilde{f}^{(a, b, c, d, 0,0)}$ $\left.\left(y-y_{0}\right) \mathrm{e}^{j \omega_{0}\left(y-y_{0}\right)}\right)$.

Hence from relation (14) it can be seen that the OLCT performs the most general linear inhomogeneous linear mapping of the time-frequency domain [3]:

$$
\left(\begin{array}{c}
y \\
\omega_{y}
\end{array}\right)=\left(\begin{array}{ll}
a & b \\
c & d
\end{array}\right)\left(\begin{array}{c}
x \\
\omega_{x}
\end{array}\right)+\left(\begin{array}{c}
y_{0} \\
\omega_{0}
\end{array}\right)
$$

which is recognized as an affine mapping. With only four parameters $(a, b, c, d)$ in Eq. (14) a homogeneous linear mapping is obtained, representing an LCT. The other two parameters $\left(y_{0}, \omega_{0}\right)$ perform an offset in the time-frequency domain, generalizing the LCT to include transforms and responses of systems that perform frequency modulation and time delay.

\subsection{The inverse OLCT}

The inverse of the mapping (15) is given by:

$$
\left(\begin{array}{c}
x \\
\omega_{x}
\end{array}\right)=\left(\begin{array}{cc}
d & -b \\
-c & a
\end{array}\right)\left(\begin{array}{c}
y \\
\omega_{y}
\end{array}\right)+\left(\begin{array}{c}
b \omega_{0}-d y_{0} \\
c y_{0}-a \omega_{0}
\end{array}\right),
$$

implying that the inverse of an OLCT with parameters $\mathbf{A}$ is given (up to a normalization factor) by an OLCT with parameters $\mathbf{A}^{-1}=\left(d,-b,-c, a, b \omega_{0}-d y_{0}\right.$, $\left.c y_{0}-a \omega_{0}\right)$ as mentioned in Sect. 2. The exact inverse OLCT expression is given by [2]:

$$
\begin{aligned}
f(x) & =C \int_{-\infty}^{\infty} \tilde{f}^{\mathbf{A}}(y) h_{\mathbf{A}^{-1}}(x, y) \mathrm{d} x, \\
C & =\mathrm{e}^{\frac{j}{2}\left(c d y_{0}^{2}-2 a d y_{0} \omega_{0}+a b \omega_{0}^{2}\right)} .
\end{aligned}
$$

4.3 Interpretation of the sampling theorem in the time-frequency domain

A heuristic interpretation of the sampling and reconstruction Theorem 1 in the Wigner domain is as follows. Suppose that the support of the WVD of $f(x)$ is as plotted in Fig. 1a. The OLCT performs an affine mapping (15) of the Wigner domain as shown in Fig. 1b. From simple geometrical considerations it can be seen that if $f(x)=0 x \notin\left[-\frac{B_{x}}{2}, \frac{B_{x}}{2}\right]$ then the WVD of $\tilde{f}^{\mathbf{A}}(y)$ vanishes outside the parallel lines $\omega_{y}=\alpha\left(y-y_{0}\right)+\omega_{0}-\Omega / 2$ and $\omega_{y}=\alpha\left(y-y_{0}\right)+\omega_{0}+\Omega / 2$ where $\alpha=\frac{d}{b}$ and $\Omega=\frac{B_{x}}{|b|}$. A multiplication of $\tilde{f}^{\mathbf{A}}(y)$ by the term $\mathrm{e}^{-\frac{j}{2 b}\left[d y^{2}-2 y\left(d y_{0}-b \omega_{0}\right)\right]}$ centralizes the WVD around $\omega_{y}=0$ and performs a $\omega_{y}$-shearing ("de-chirping") in the Wigner domain [20] as shown in Fig. 1c; as a result, WVD is nonzero only for the frequency range $\omega_{y} \in[-\Omega / 2,-\Omega / 2]$. This infers that the function with the WVD of Fig. 1c is bandlimited in the frequency range $[-\Omega / 2,-\Omega / 2]$ hence, according to Shannon-Whittaker-Kotel'nikov sampling theory [1], it can be completely recovered from its samples at intervals $\Delta_{y}=\frac{2 \pi}{\Omega}$ using Whittaker's cardinal (sinc) functions [21]. Then, by applying an inverse $\omega_{y}$-shearing and performing an inverse frequency shift by multiplication by $\mathrm{e}^{\frac{j}{2 b}\left[d y^{2}-2 y\left(d y_{0}-b \omega_{0}\right)\right]}$, the continuous $\tilde{f}^{\mathbf{A}}(y)$ can be obtained. Following the geometrical description of the OLCT in the Wigner domain (Fig. 1a, b) together with the relations $\alpha=\frac{d}{b}$ and $\Omega=\frac{B_{x}}{|b|}$ we can derive the following corollary from Theorem 1 .

Corollary 1 If a function has a compact support in the timefrequency Wigner domain such that its $W V D W_{f}(t, \omega)$ is zero for frequencies $\omega \notin\left[\alpha\left(t-t_{0}\right)+\omega_{0}-\Omega / 2, \alpha\left(t-t_{0}\right)+\right.$ $\left.\omega_{0}+\Omega / 2\right]$ for every time $t$, where $\alpha, t_{0}, \omega_{0}$ are real finite constants, then it can be completely reconstructed from its samples at points spaced $T=\frac{2 \pi}{\Omega}$ apart. The interpolation formula is then given by:

$$
\begin{aligned}
f(t)= & \mathrm{e}^{\frac{j}{2}\left[\alpha t^{2}-2 t\left(\alpha t_{0}-\omega_{0}\right)\right]} \sum_{n \notin Z} f(n T) \frac{\sin \left[\pi\left(\frac{t}{T}-n\right)\right]}{\pi\left(\frac{t}{T}-n\right)} \\
& \times \mathrm{e}^{-\frac{j}{2}\left[\alpha(n T)^{2}-2 n T\left(\alpha t_{0}-\omega_{0}\right)\right]} .
\end{aligned}
$$

In Corollary 1 we have changed the notations of the parameter and Fourier reciprocal from $\left(y, \omega_{y}\right)$ to $(t, \omega)$ to make it clearer in the context of common time signals. 
Fig. 1 a The support in the Wigner domain of function $f(x)$. b The WVD of the OLCT is an affine transform of a. c A Fourier band limited function is obtained by multiplying by $\mathrm{e}^{-\frac{j}{2 b}\left[d y^{2}-2 y\left(d y_{0}-b \omega_{0}\right)\right]}$

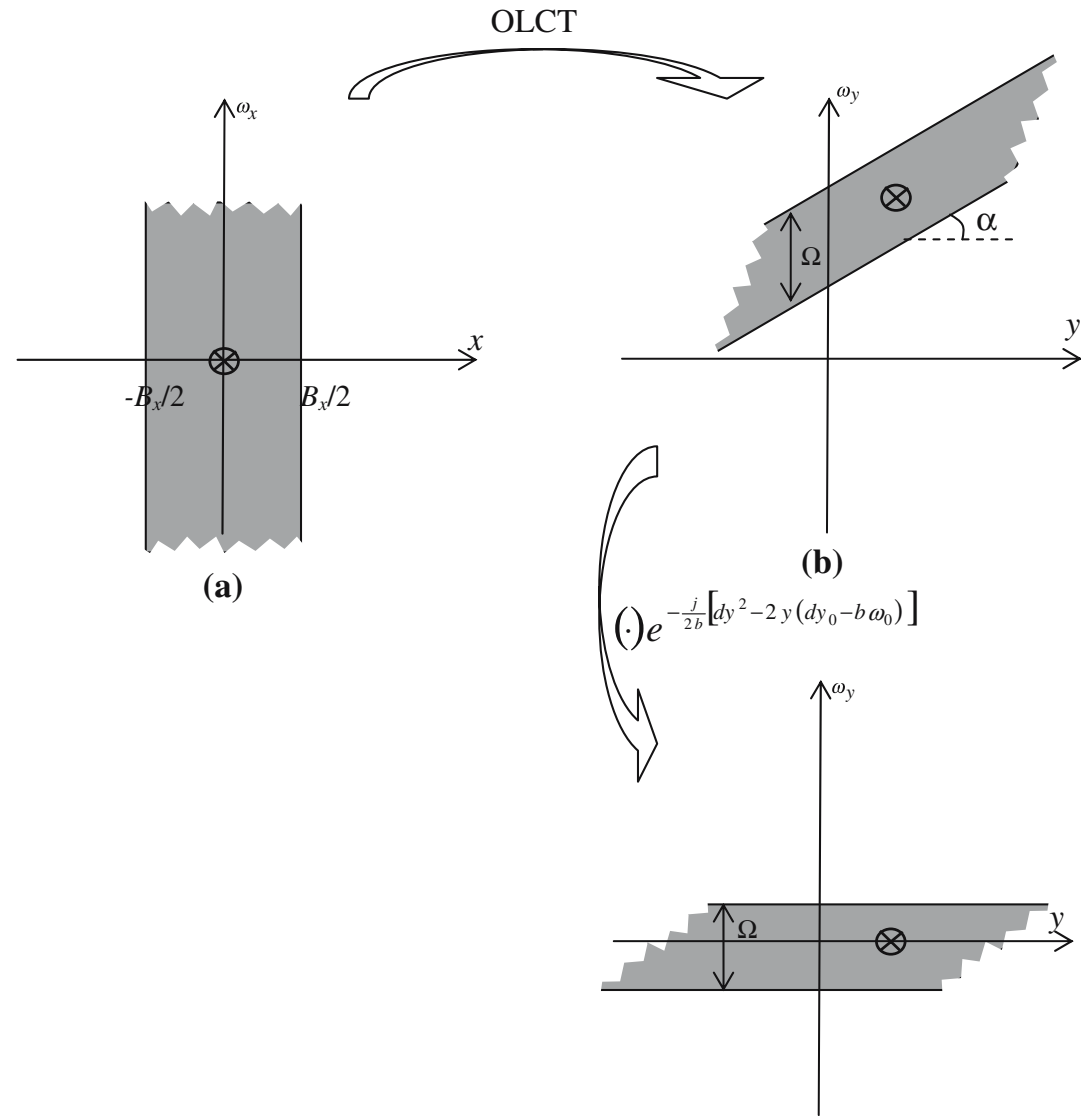

(c)
Proof Note that the conditions of the corollary are that of a function with Wigner domain as shown in Fig. 1b. This function is the OLCT with parameters $\mathbf{A}=(a, b, c, d$, $\left.y_{0}, \omega_{0}\right), b \neq 0$ of a function $f(x)$ exactly as considered in Theorem 1. Therefore, by substituting relations $\Omega=\frac{B_{x}}{|b|}$ and $\alpha=\frac{d}{b}$ in the sampling condition of Theorem 1 and in Eq. (3) we obtain the sampling condition of the corollary and the interpolation formula (18).

Loosely speaking, Corollary 1 states that if a function has a maximum instant (double sided) bandwidth of $\Omega$ with respect to some instantaneous frequency $\alpha\left(t-t_{0}\right)+\omega_{0}$, then the sampling rate for lossless uniform sampling is $\frac{2 \pi}{\Omega}$ and the restoration formula is given by Eq. (18). Please note that the sampling interval depends only on $\Omega$ and not on the angle $\alpha$. In the specific case that $\alpha=0$, that is the instantaneous frequency is time independent, the maximum instant bandwidth of $\Omega$ equals to the traditional overall bandwidth, and Corollary 1 reduces to the Shannon sampling theorem.

Figure 2 illustrates some examples of common transforms, all being special cases of OLCT, as are reflected in the timefrequency domain. We assume that the signal $f(x)$ has a compact support therefore its WVD is confined in the $x$ direction as shown in Fig. 2a. Since the signal is time limited it has an infinite Fourier bandwidth. However, for illustrative purposes we will assume that the signal is $\varepsilon_{\omega}$-concentrated [22] in the range $\omega_{x} \in\left[-\frac{W^{\prime}}{2}, \frac{W^{\prime}}{2}\right]$, i.e., the portion of signal energy left out of this range is $\varepsilon_{\omega}$.. If we choose $\varepsilon_{\omega}$ sufficiently small we may refer to $W^{\prime}$ as the "effective (double sided) bandwidth" of $f(x)$. Figure $2 \mathrm{~b}$ depicts the WVD support of the OLCT of $f(x)$ with $\mathbf{A}=\{\cos \varphi, \sin \varphi,-\sin \varphi$, $\cos \varphi, 0,0\}$ which is recognized as a FRFT. It can be seen that the FRFT performs a rotation of the WVD support of $f(x)$. The instantaneous frequency of $\tilde{f}^{\mathbf{A}}(y)$ is along the line $\omega_{y}=\frac{1}{\tan \varphi} y$ (dashed) and the instantaneous bandwidth is $\Omega=\frac{B_{x}}{|\sin \varphi|}$. Hence, from Corollary 1 we have the sampling condition: $\Delta_{y} \leq \frac{2 \pi}{B_{x}}|\sin (\varphi)|$. The same sampling condition together with the interpolation formula (3) was derived previously using different analyses in [11-14,16]. A FRFT with $\varphi=\pi / 2$ becomes a FT. With a $\pi / 2$ rotation of the WVD, the instantaneous bandwidth is $\Omega=B_{x}$ yielding the sampling condition $\Delta_{y} \leq \frac{2 \pi}{B_{x}}$. If we replace the roles of $f(x)$ and $\tilde{f}^{A}(y)$ so that we refer to $\tilde{f}^{A}(y)$ as the signal in the time domain and to $f(x)$ as its Fourier pair, then it is 
Fig. 2 a The support of $f(x)$ in the Wigner domain. b-f

Examples of the support of the

WVD of several OLCT of $f(x)$

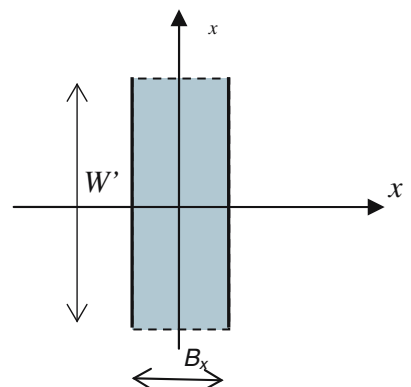

(a)

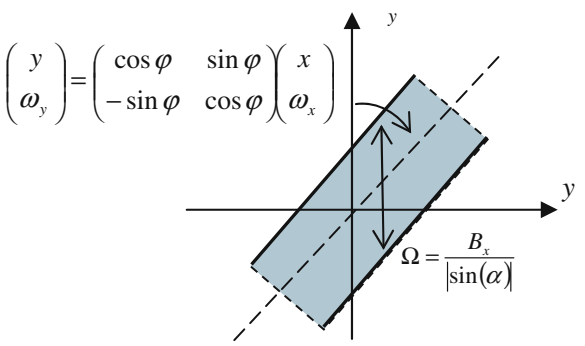

(b)

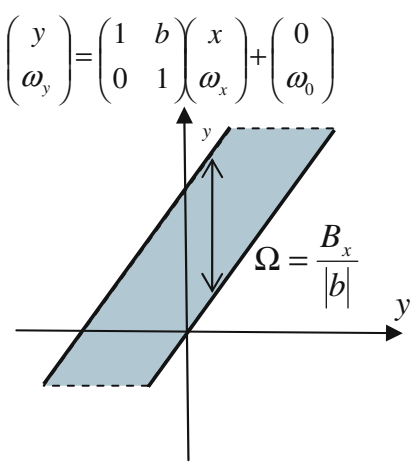

(c)

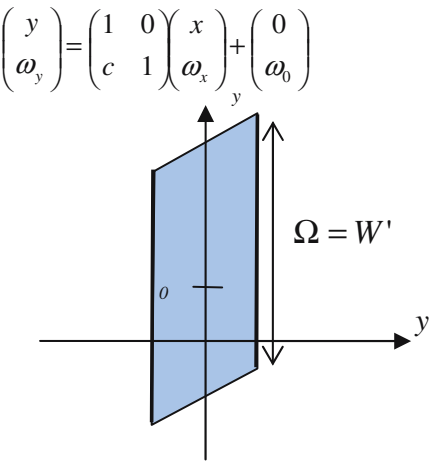

(e)

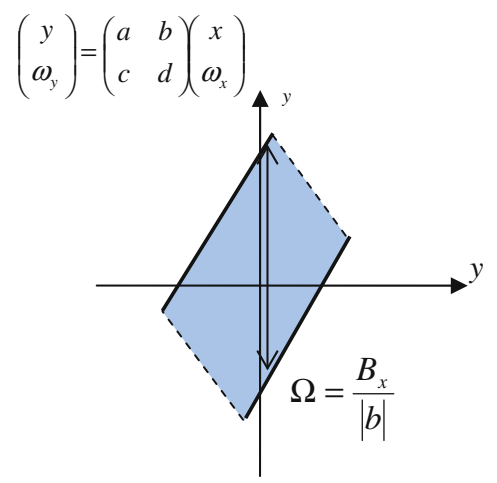

(f) evident that $B_{x}$ in Fig. 2a is the Fourier bandwidth of $\tilde{f}^{A}(y)$. Consequently, the Nyquist rate [1] is $\Delta_{y} \leq \frac{2 \pi}{B_{x}}$, exactly as indicated by Corollary 1 . Figure $2 \mathrm{c}$ shows the WVD support of the OLCT of $f(x)$ with $\mathbf{A}=\left\{1, b, 0,1,0, \omega_{0}\right\}$. According to Corollary 1 the sampling condition is $\Delta_{y} \leq$ $\frac{2 \pi}{B_{x}}|b|$. Note that for $\Omega=\frac{B_{x}}{|b|}<W^{\prime}$ this sampling condition is looser than the Nyquist condition $\left(\Delta_{y} \leq \frac{2 \pi}{W^{\prime}}\right)$. Consequently, recovering the signal from samples below the Nyquist rate is possible [16]. Figure 2d shows the WVD support of time shifted and modulated $f(x)$, corresponding to an OLCT with $\left(1,0,0,1, y_{0}, \omega_{0}\right)$. The instantaneous frequency is time independent $(\alpha=0)$ and equals $\omega_{0}$. The instantaneous bandwidth is the same as the effective bandwidth; i.e., $\Omega=W^{\prime}$. Consequently, according to Corollary 1 , the sampling interval is $\Delta_{y} \leq \frac{2 \pi}{W^{\prime}}$ which is the same as indicated by Nyquist criterion for $f(x)$. Thus we see that time shifting and frequency modulation do not affect the sampling condition. In Fig. 2e the WVD support of the OLCT of $f(x)$ with $\mathbf{A}=\left\{1,0, c, 1,0, \omega_{0}\right\}$ is shown. This OLCT represents a multiplication of $f(x)$ by the quadrature phase function $\mathrm{e}^{j \frac{c}{2} x^{2}+j \omega_{0} x}$. The instantaneous bandwidth and the sampling condition are the same as in Fig. 2d. However, note that the interpolation formula (18) differs from that for the signal in Fig. 2 because the instantaneous frequency is time dependent $(\alpha \neq 0)$. Finally, in Fig. 2f the support of $\tilde{f}^{\mathbf{A}}(y)$ with $\mathbf{A}=\{a, b, c, d, 0,0\}$ representing a general LCT is shown. It can be seen that the WVD support performs a homogeneous affine transform. According to Theorem 1, the 
sampling condition is $\Delta_{y} \leq \frac{2 \pi}{B_{x}}|b|$ which can be also obtained from Corollary 1 with $\Omega=\frac{B_{x}}{|b|}$. This sampling condition is the same as derived using a different analysis in [16].

\section{Sampling condition and the uncertainty principle for OLCT pairs}

Note that the sampling interval $\Delta_{y} \leq \frac{2 \pi}{B_{x}}|b|$ given in Theorem 1 is proportional to the OLCT transform parameter $b$. Loosely speaking, this implies that the concentration of the OLCT of a signal $f(x)$ with a compact support (or more generally $\varepsilon_{x}$-concentrated [22] in the $x$ domain) is dictated by the parameter $b$. An OLCT with large $b$ causes a large spread of the signal, and vice versa. This property can be clearly seen from geometrical considerations in the time-frequency domain. It is also interesting to note that this fact is in concordance with the uncertainty relation for OLCT pairs given by:

$\sigma_{f(x)}^{2} \sigma_{\tilde{f}(y)}^{2} \geq \frac{|b|^{2}}{4}$

where $\sigma_{f(x)}^{2}$ and $\sigma_{\tilde{f}(y)}^{2}$ are the variances of $f(x)$ and $\tilde{f}^{\mathbf{A}}(y)$, respectively

$\sigma_{f(x)}^{2}=\frac{1}{\|f(x)\|^{2}} \int_{-\infty}^{\infty}(x-u)^{2}|f(x)|^{2} \mathrm{~d} x$,

$\sigma_{\tilde{f}(y)}^{2}=\frac{1}{\left\|\tilde{f}^{\mathbf{A}}(y)\right\|^{2}} \int_{-\infty}^{\infty}(y-\xi)^{2}\left|\tilde{f}^{\mathbf{A}}(y)\right|^{2} \mathrm{~d} y$,

and $u$ and $\xi$ are the average moments respectively

$$
\begin{aligned}
& u=\frac{1}{\|f(x)\|^{2}} \int_{-\infty}^{\infty} x|f(x)|^{2} \mathrm{~d} x, \\
& \xi=\frac{1}{\left\|\tilde{f}^{\mathbf{A}}(y)\right\|^{2}} \int_{-\infty}^{\infty} y\left|\tilde{f}^{\mathbf{A}}(y)\right|^{2} \mathrm{~d} y .
\end{aligned}
$$

The proof of relation (19) is given in Appendix A. Relation (19) can be used to study the joint localization of $f(x)$ and $\tilde{f}^{\mathbf{A}}(y)$ in a similar way as to that in which the HeisenbergWeyl uncertainty principle is commonly used to study localization in time-frequency analysis (see for example [23]). From relation (19) we see that for a given $\sigma_{f(x)}$, the spread $\sigma_{\tilde{f}(y)}$ of $\tilde{f}^{\mathbf{A}}(y)$ is dependent on $b$, implying that the concentration of the OLCT of a signal with a given spread in $\sigma_{f(x)}$ the $x$ domain is dependent only on the OCLT parameter $b$.

Please note that the uncertainty relation (19) generalizes other uncertainty relations. In the particular cases of an OLCT with parameters $\mathbf{A}=\{0,-1,-1,0,0,0\}$, implementing a FT, relation (19) reduces to the well known
Heisenberg-Weyl uncertainty relation. We see also that the Heisenberg-Weyl uncertainty relation holds for any OLCT pair with $\mathbf{A}=\left(a, \pm 1, c, d, y_{0}, \omega_{0}\right)$. An OLCT with parameters $\mathbf{A}=\{\cos \varphi, \sin \varphi,-\sin \varphi, \cos \varphi, 0,0\}$ relation (19) reduces to the uncertainty principle for FRFT pairs [24].

\section{Simulation example}

The sampling theorem is illustrated with the OLCT pair $f(x)$ and $\tilde{f}^{\mathbf{A}}(y)$ shown in Fig. $3 \mathrm{a}$ and $\mathrm{b}$, respectively. It can be seen that the function $f(x)$ has compact support $x \in[-1 / 2,1 / 2]$. This function is OLCTed with parameters $\mathbf{A}=[1,0.25,0,1,0,2]$ to obtain $\tilde{f}^{\mathbf{A}}(y)$ shown in Fig. $3 \mathrm{~b}$. The time-frequency representation of $f(x)$ and $\tilde{f}^{\mathbf{A}}(y)$ is as depicted in Fig. 2a and c, respectively. A OLCT with parameter set $\mathbf{A}$ of this form, with possibly different $b$ and $\omega_{0}$ values, can be encountered in various physical systems. For instance, this type of transform can represent the scalar optical diffraction of the field propagating from a double slit that is captured with a tilted planar sensor. It can also represent the echo received from two scatterers spatially distributed as obtained with a radar system that uses time pulse compression. This type of transform can be found also in digital holography, sonar systems, and atomic interferometry.

The OLCTed signal $\tilde{f}^{\mathbf{A}}(y)$ was uniformly sampled with sampling period $\Delta_{y}=1$. The real and imaginary parts of the samples of $\tilde{f}^{\mathbf{A}}(y)$ are represented by the stem graphs in Fig. $3 \mathrm{c}$ and d, respectively. The sampled signal has been interpolated using Eq. (3) to obtain the signals with real and imaginary parts shown in Fig. 3c and d, respectively. It can be seen that the reconstructed signal denoted by dashed lines is almost undistinguished from the original signal denoted by continuous lines. It is noteworthy that the sampling interval $\Delta_{y}$ is larger than the width of the pulses and the gap between them in the original signal shown in Fig. 3a. However, this interval suffices to maintain the signal information in the transformed domain due to time spreading shown in Fig. 2c.

\section{Conclusions}

In this paper the common Shannon's sampling theorem for signals band limited in Fourier domain is generalized to signals band limited in OLCT domain. The sampling theorem can be applied to a large class of signals and system outputs. The sampling theorem developed can be regarded as a unified and extended version of previously developed sampling theorems for particular cases of the OLCT. In terms of timefrequency representation, the presented sampling theorem appeals to all signals that an inhomogeneous affine mapping 
Fig. 3 a Function $f(x)$ with compact support, $\mathbf{b}$ the absolute value $\left|\tilde{f}^{\mathbf{A}}(y)\right|$ of the OLCT of $f(x)$ with parameters $\mathbf{A}=[1,0.25,0,1,0,2]$; c, $\mathbf{d}$ real and imaginary parts respectively, of $\tilde{f}^{\mathbf{A}}(y)$ (continuous line), of the samples of $\tilde{f}^{\mathbf{A}}(y)$ (stem graph), and of the signal reconstructed from its samples (dashed line). For illustration purposes the real and imaginary parts of $\tilde{f}^{\mathbf{A}}(y)$ are shown in the reduced range $-4.5<y<4.5$
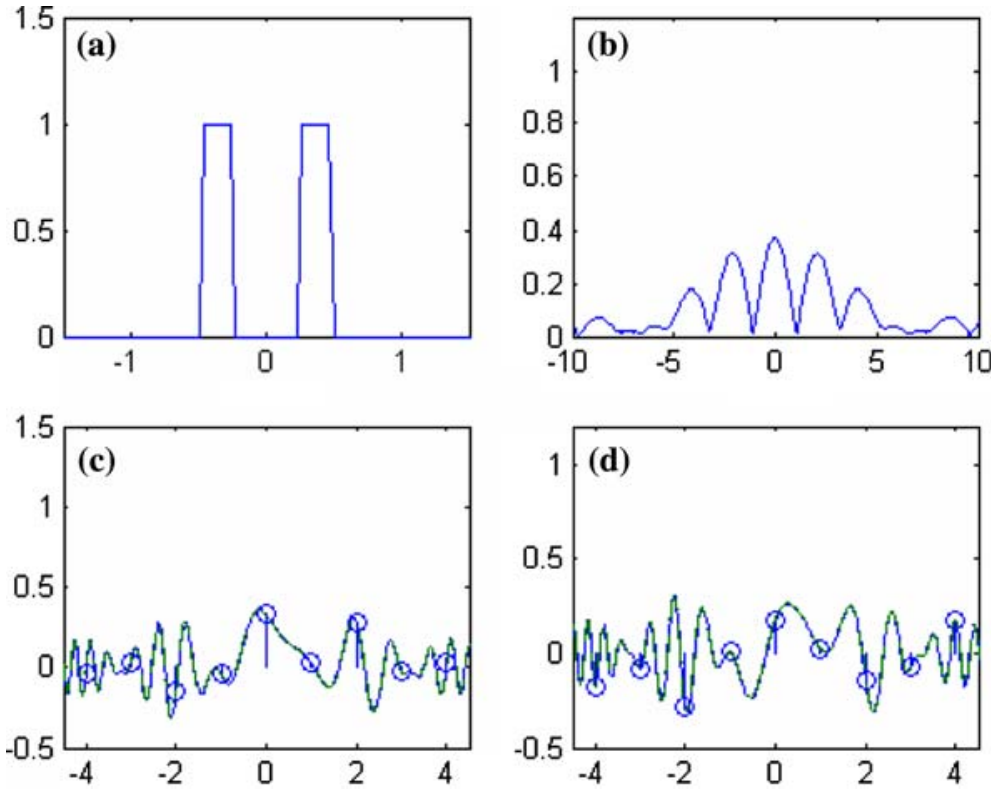

of their time-frequency domain yields a time limited signal. In this work we also present the uncertainty principle for OLCT pairs which generalizes the well known HeisenbergWeyl uncertainty principle. It is shown that the same transform parameter that governs the sampling rate also dictates the uncertainty relation.

\section{Appendix}

Proof of the uncertainty relation for OLCT pairs

Observe that by substituting Eq. (4) in expression (21) we have

$$
\begin{aligned}
\sigma_{\tilde{f}(y)}^{2} & =\frac{1}{\left\|\tilde{f}^{\mathbf{A}}(y)\right\|^{2}} \int_{-\infty}^{\infty}(y-\xi)^{2}\left|\tilde{f}^{\mathbf{A}}(y)\right|^{2} \mathrm{~d} y \\
& =\frac{1}{\|G(y)\|^{2}} \int_{-\infty}^{\infty}(y-\xi)^{2}|G(y)|^{2} \mathrm{~d} y \equiv \sigma_{G(y)}^{2}
\end{aligned}
$$

where $\sigma_{G(y)}^{2}$ is the variance of $G(y)$. Now let us consider the scaled function $G(r)$ where $r=y / b$. Its variance is

$\sigma_{G\left(\frac{y}{b}\right)}^{2}=\frac{1}{|b|^{2}} \sigma_{G(y)}^{2}=\frac{1}{|b|^{2}} \sigma_{\tilde{f}(y)}^{2}$.

From (5) we see that $G(r)$ is the Fourier transform of $g(x)=f(x) \mathrm{e}^{\frac{j}{2 b}\left(a x^{2}+2 y_{0} x\right)}$. Hence, from the HeisenbergWeyl uncertainty principle for Fourier pairs $G(r)$ and $g(x)$ we have $\sigma_{g(x)}^{2} \sigma_{G(r)}^{2} \geq \frac{1}{4}$ which, together with (A2) yields

$\sigma_{g(x)}^{2} \frac{1}{|b|^{2}} \sigma_{\tilde{f}(y)}^{2} \geq \frac{1}{4}$, where $\sigma_{g(x)}^{2}$ denotes the variance of $g(x)$. Note that $|g(x)|=$ $|f(x)|$ therefore the functions $g(x)$ and $f(x)$ have the same variance, thus replacing $\sigma_{g(x)}^{2}$ by $\sigma_{f(x)}^{2}$ in (A3) yields (19).

\section{References}

1. Shannon, C.E.: Communication in the presence of noise. Proc. IRE 37, 447-457 (1949)

2. Pei, S.C., Ding, J.J.: Eigenfunctions of the offset Fourier, fractional Fourier, and linear canonical transforms. J. Opt. Soc. Am. A 20, 522-532 (2003)

3. Abe, S., Sheridan, J.T.: Optical operations on wave functions as the Abelian subgroups of the special affine Fourier transformation. Opt. Lett. 19, 1801-1803 (1994)

4. Moshinsky, M., Quesne, C.: Linear canonical transformations and their unitary representations. J. Math. Phys. 12, 1772-1783 (1971)

5. Pei, S.C., Ding, J.J.: Eigenfunctions of linear canonical transform. IEEE Trans. Acoust. Speech. Sig. Process. 50, 11-26 (2002)

6. Wolf, K.R.: Integral Transforms in Science and Engineering, Chap. 9,10. Plenum Press, New York (1979)

7. Almeida, L.B.: The fractional Fourier transform and time frequency representation. IEEE Trans. Sig. Process. 42, 30843091 (1994)

8. Ozaktas, H.M., Kutay, M.A., Zalevsky, Z.: The Fractional Fourier Transform with Applications in Optics and Signal Processing. Wiley, New York (2000)

9. Papoulis, A.: Pulse compression, fiber communication, and diffraction: a unified approach. J. Opt. Soc. Am. A 11, 3-13 (1994)

10. Pei, S.C., Ding, J.J.: Generalized eigenvectors and fractionalization of offset DFTs and DCTs. IEEE Trans. Sig. Proc. 52(7), 20322046 (2004)

11. Pei, S.C., Yeh, M.H., Luo, T.L.: Fractional Fourier series expansion for finite signals and dual extension to discrete-time fractional Fourier transform. IEEE Trans. Sig. Proc. 47(10), 2883 2888 (1999)

12. Xia, X.G.: On bandlimited signals with fractional Fourier transform. IEEE Sig. Proc. Lett. 3(3), 72-74 (1996) 
13. Zayed, A.I.: On the relationship between the Fourier and fractional Fourier transforms. IEEE Sig. Proc. Lett. 3(12), 310-311 (1996)

14. Candan, C., Ozaktas, H.M.: Sampling and series expansion theorems for fractional Fourier and other transforms. Sig. Process. 83(11), 2455-2457 (2003)

15. Gori, F.: Fresnel transform and sampling theorem. Opt. Com. 39, 293-297 (1981)

16. Stern, A.: Sampling of linear canonical transformed signals. Sig. Process. 87, 1421-1425 (2006)

17. Kramer, H.P.: A generalized sampling theorem. J. Math. Phys. 38, 68-72 (1959)

18. Wigner, E.P.: On the quantum correlation for thermodynamic equilibrium. Phys. Rev. 40, 749-759 (1932)
19. Cohen, L.: Time-frequency distributions-a review. Proc. IEEE 77, 941-981 (1989)

20. Pei, S.C., Ding, J.J.: Relations between fractional operations and time-frequency distributions, and their applications. IEEE Trans. Sig. Proc. 49, 1638-1655 (2001)

21. Whittaker, M.: The Fourier theory of the cardinal functions. Proc. Math. Soc. Edinburgh 1, 169-176 (1929)

22. Grochenig, K.: Foundation of time-frequency analysis. Birkhauser, Boston (2001)

23. Mallat, S.: A wavelet tour of signal processing, 2nd edn. Academic, San Diego, pp. 31-32 (1999)

24. Ozaktas, H.M., Aytur, O.: Fractional Fourier domains. Sig. Process. 46, 119-124 (1995) 